\title{
Comparison of Alarm Strategies for Continuous 12-Lead ST-Segment Monitoring
}

\author{
JY Wang, MC Saenz, BM Horáček* \\ Philips Medical Systems, Andover, MA, USA \\ *Dalhousie University, Halifax, Nova Scotia, Canada
}

\begin{abstract}
Several alarm detection strategies for real-time STsegment monitoring (including detection using any single lead, any two leads, any two contiguous leads, any three leads, and STindex, computed as the sum of the absolute ST values from three quasi-orthogonal leads) have been evaluated using a 12-lead ST database generated from the Dalhousie PTCA database. The database includes 95 patients with a total of 202 balloon occlusions.

For all the occluded vessels combined, the detection sensitivity using any single lead for ST-alarm detection decreases from $84.7 \%$ to $74.8 \%$ when the threshold is increased from $1 \mathrm{~mm}$ to $1.5 \mathrm{~mm}$. For alarm detection using any two leads, two contiguous leads, and three leads, the results using a 1-mm threshold are 79.7\%, $78.7 \%$, and $77.2 \%$, respectively. The two-lead performance results can be improved to $87.1 \%$ for any two leads and to $84.2 \%$ for any two contiguous leads if a lower threshold of $0.8 \mathrm{~mm}$ is used. For all the possible three-lead combinations, the STindex computed using leads III, V2, and V5 shows the highest correlation to the sum of the absolute ST values from all 12 leads. A detection sensitivity of $85.2 \%$ can be obtained using STindex with a threshold of $1.5 \mathrm{~mm}$.
\end{abstract}

\section{Introduction}

Ischemia detection has always been an important component in identifying and managing patients with coronary artery disease. Advances in management of patients with acute MI, such as early reperfusion with thrombolytic therapy and revascularization with a PTCA procedure, have made continuous non-invasive detection of ischemia even more important. The emphasis has shifted from detecting and diagnosing coronary artery disease to on-line monitoring and treatment of evolving ischemia. Although monitoring ECG for ST-segment deviation is not the most sensitive and specific technique for myocardial ischemia detection, it remains the only practical technique for continuous non-invasive monitoring of ischemic episodes.

The location of the monitoring electrodes and the direction and magnitude of the ST-segment changes indicate the region of the heart at risk, and the possible extent of the damage. The probability of detecting ischemic episodes and locating them increases with the number of leads employed. Today's monitors are capable of providing up to 12 ECG leads for continuous monitoring. The presence of ST-segment deviation of 1 $\mathrm{mm}(0.1 \mathrm{mV})$ or greater in at least one lead is the current standard for ischemia detection [1,2]. While this threshold is highly sensitive, it also has the potential of generating many false alarms when used in a real-time monitoring environment [3], especially when the number of ECG leads being monitored is large, e.g., when 12-lead ECG is employed for continuous monitoring.

In order to reduce the number of false alarms, several ST-alarm strategies have been implemented [2,4], including using higher detection thresholds, and requiring multiple leads to exceed the detection thresholds. However, these alarm strategies have not been directly compared on the basis of their sensitivity in detecting STsegment changes. The objective of this study is to compare several multi-lead alarm strategies by using a common ST database for their ability in detecting STsegment changes. The present study also evaluates whether the single-valued ST parameter, STindex, computed as the sum of the absolute ST values from three orthogonal leads, can be used to achieve the same detection sensitivity obtained when all 12 leads are used.

\section{Method}

12-lead ST Database - The 12-lead ST database used in the present study is generated from the Dalhausie PTCA database [5]. It contains a total of 202 balloon occlusions from 95 PTCA patients with single-vessel disease. Vessels dilated included left anterior descending (LAD) coronary artery in 35 patients with 76 inflations, right coronary artery (RCA) in 37 patients with 77 inflations, and left circumflex (LCX) coronary artery in 23 patients with 49 inflations. ST-segment changes between peak 
inflation and before inflation are computed at $60 \mathrm{~ms}$ after the $\mathrm{J}$ point for the standard 12 ECG leads (Mason-Likar limb-lead placement) from the 120-lead body surface potential map recorded during the PTCA procedure.

STindex - The STindex is a single-valued parameter used for ST-segment monitoring. It is computed as the sum of the absolute ST values from three ECG leads that can best indicate the ST changes in the different locations of the heart. One such lead-set can be formed from the three orthogonal leads aVF (superior-inferior axis), V2 (anterior-posterior axis), and V5 (lateral axis) [6]. Another quasi-orthogonal lead-set considered is the threelead combination of III, V2, and V5. This lead-set is selected because it gives the highest correlation between the computed STindex and the sum of the absolute ST values obtained from all 12 leads (ST12). Figure 1 is the scatter plot of ST12 versus STindex computed from leads aVF, V2, and V5, and Figure 2 is the same plot for leads III, V2, and V5. These plots show that, as compared to the lead-set (aVF, V2, V5), the lead-set (III, V2, V5) has both a higher correlation (0.993 vs. 0.984$)$, and a higher linear regression slope (0.324 vs. 0.282).

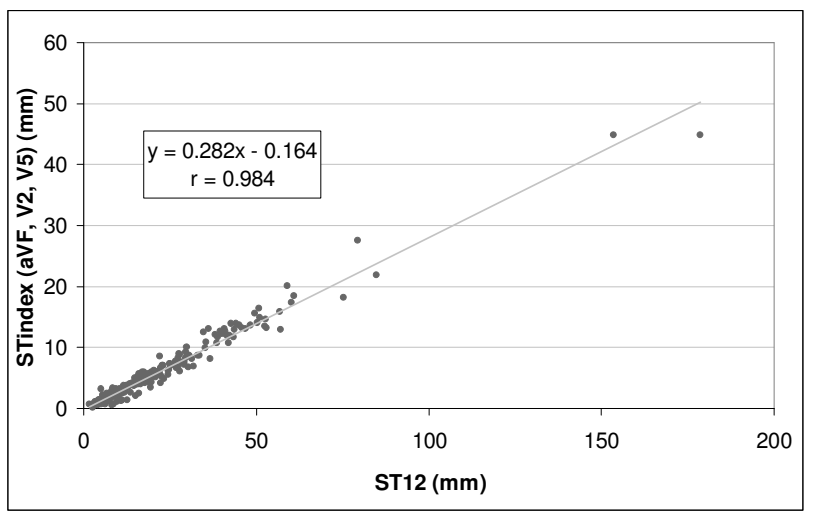

Figure 1. Scatter plot of ST12 vs. STindex computed from leads aVF, V2, and V5.

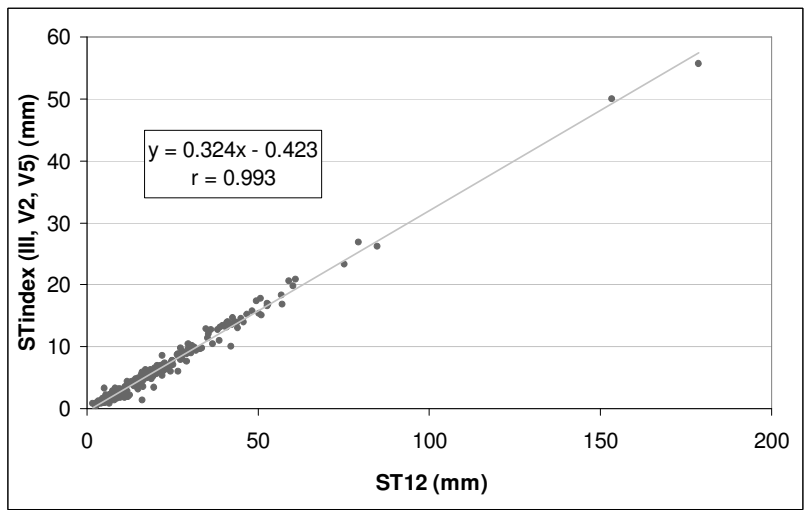

Figure 2. Scatter plot of ST12 vs. STindex computed from leads III, V2, and V5.
ST-alarm - Using the 12-lead PTCA ST database, several ST-alarm strategies are studied for their detection sensitivity performance as a function of the detection threshold. Specifically, the following alarm detection strategies based on the use of (1) any single lead, (2) any two leads, (3) any two contiguous leads, (4) any three leads, and (5) STindex are evaluated.

\section{Results}

The ST-alarm evaluation results are presented graphically in Figures 3-11. In each plot, the vertical axis represents the ST-alarm detection sensitivity (range is $50 \%$ to $100 \%$ ), and the horizontal axis represents the ST detection threshold (range is 0 to $2.0 \mathrm{~mm}$ ). Figures 3-10 show the performance results of the various ST-alarm strategies studied. In Figures 3-5 and 8-10, the results are presented separately for each of the three occluded vessels (LAD, RCA, and LCX), and all three vessels combined. The two-lead results are shown in Figures 6 and 7 with independent detection thresholds. However, to

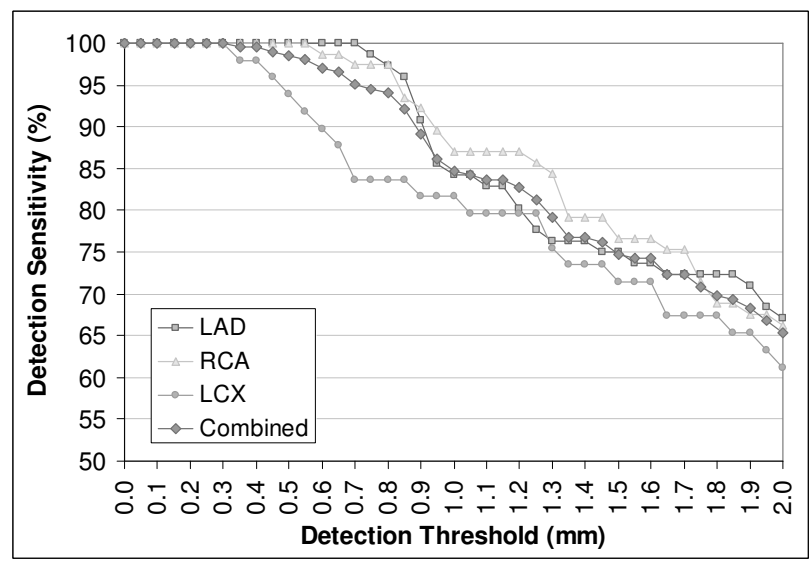

Figure 3. Detection sensitivity as a function of the detection threshold using any single lead.

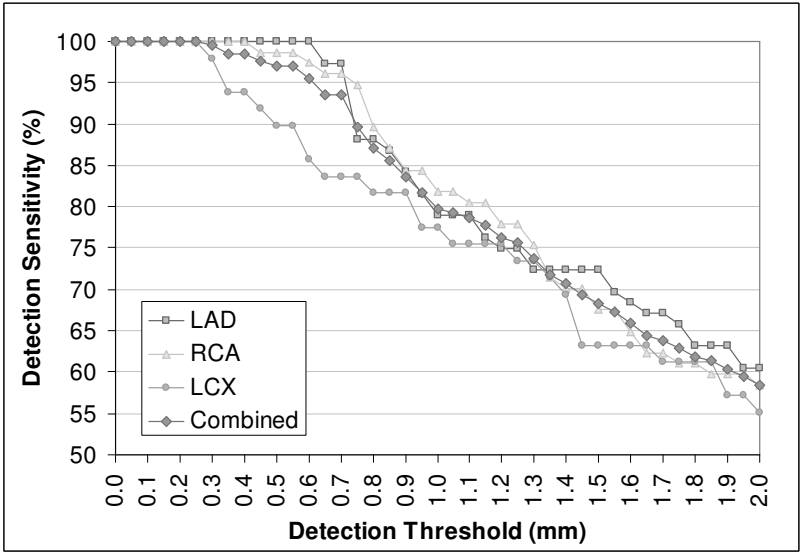

Figure 4. Detection sensitivity as a function of the detection threshold using any two leads (same thresholds are used for both leads). 


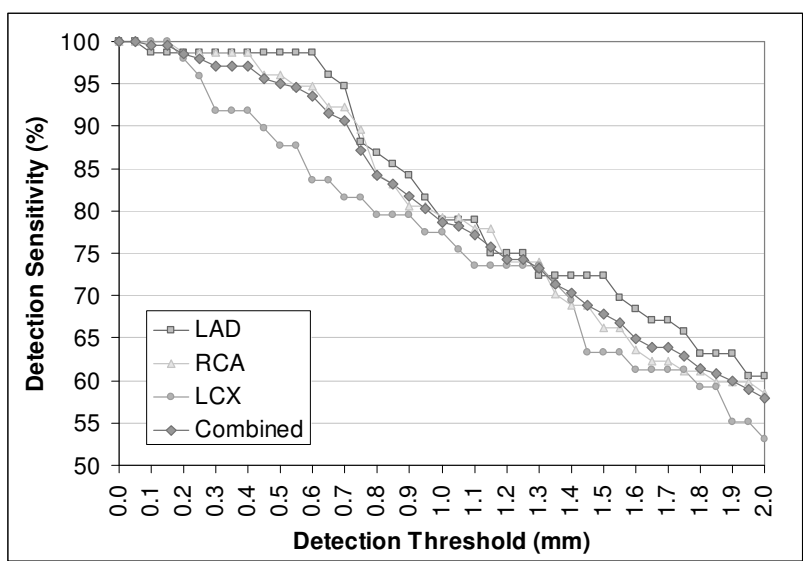

Figure 5. Detection sensitivity as a function of the detection threshold using any two contiguous leads (same thresholds are used for both leads).

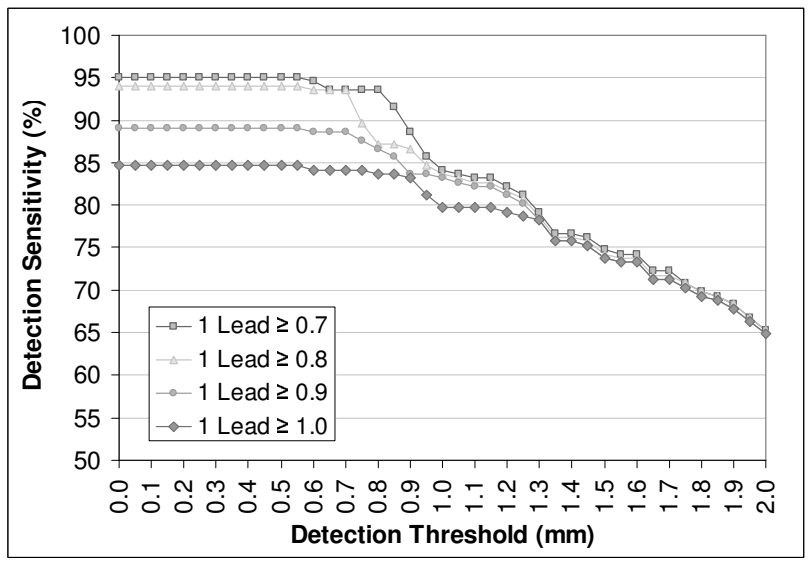

Figure 6. Detection sensitivity as a function of the detection threshold using any two leads (with independent thresholds).

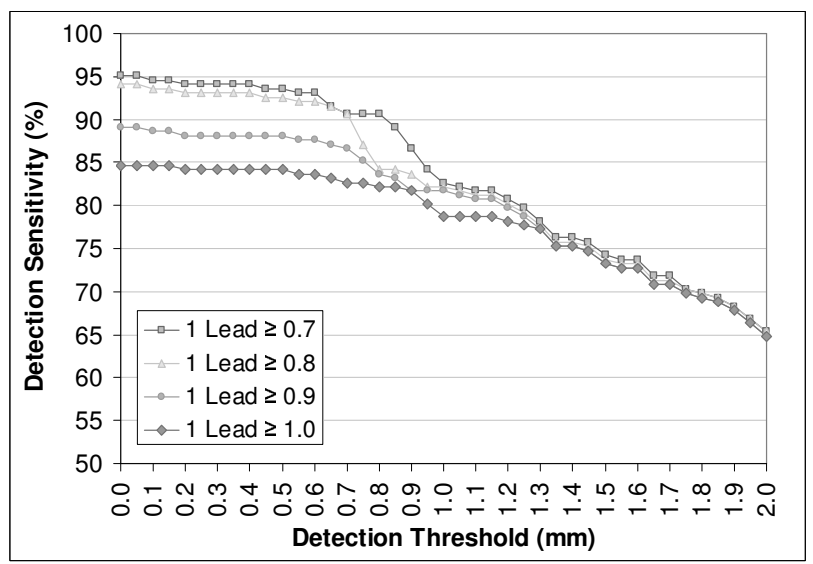

Figure 7. Detection sensitivity as a function of the detection threshold using any two contiguous leads (with independent thresholds).

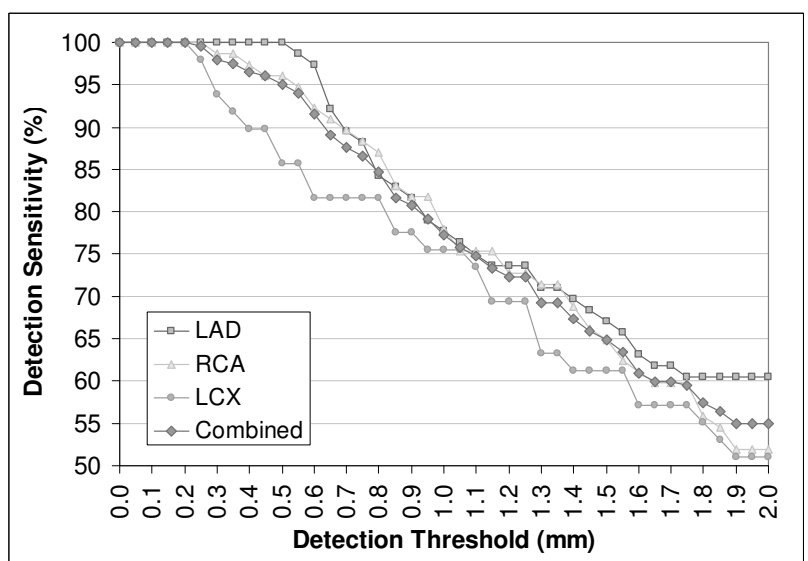

Figure 8. Detection sensitivity as a function of the detection threshold using any three leads.



Figure 9. Detection sensitivity as a function of the detection threshold using STindex computed from leads aVF, V2, and V5.

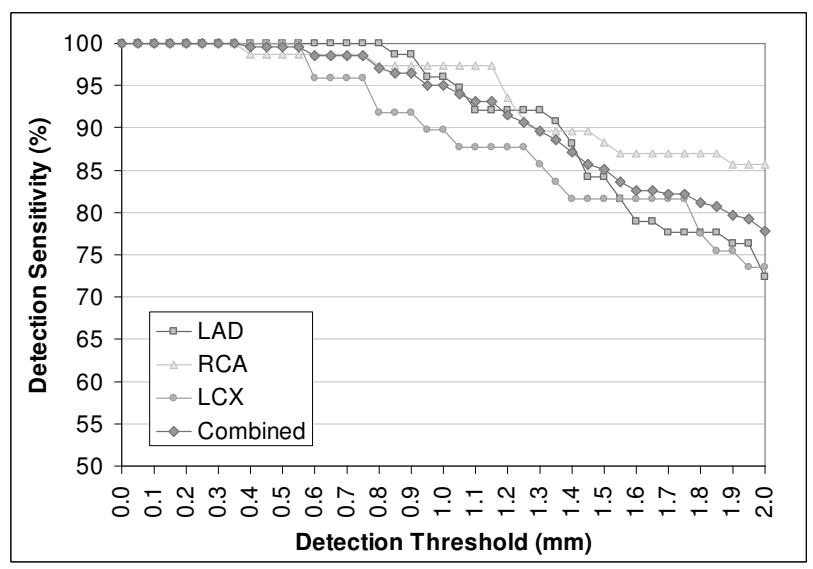

Figure 10. Detection sensitivity as a function of the detection threshold using STindex computed from leads III, V2, and V5. 


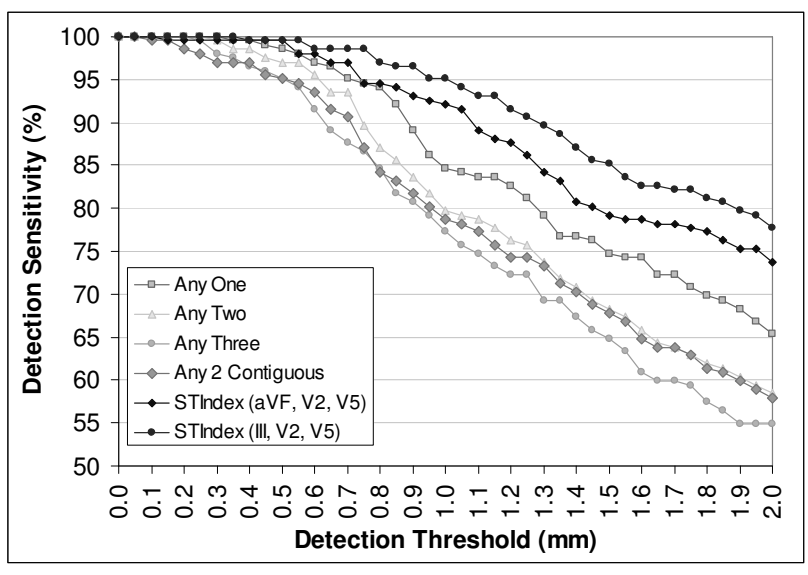

Figure 11. Detection sensitivity performance summary for all the evaluated ST-alarm detection strategies.

make the graph readable, only four relevant threshold values $(0.7$ to 1.0$)$ are shown for one of the two leads. Figure 11 is the detection performance summary plot for all the evaluated ST-alarm strategies.

Any single lead (Figure 3) - This figure shows that the detection sensitivity drops rapidly as the detection threshold increases (from $84.7 \%$ at $1 \mathrm{~mm}$ to $74.8 \%$ at 1.5 $\mathrm{mm}$ ). This quick drop of detection sensitivity makes the use of a higher threshold for false alarm reduction not a very desirable solution.

Any two leads with the same thresholds (Figure 4) The result shows that, on the average, there is a drop of about $5 \%$ in sensitivity when two leads are required for an ST-alarm. The sensitivity can be improved by using a slightly lower threshold. A sensitivity of $85.6 \%$ can be obtained if a detection threshold of $0.85 \mathrm{~mm}$ is used for both leads.

Any two contiguous leads with the same thresholds (Figure 5) - This figure shows that there is an additional small reduction in detection sensitivity (about $2 \%$ on the average) when two contiguous leads are required for an ST-alarm. However, this reduction is not considered very significant.

Any two leads or two contiguous leads with independent thresholds (Figures 6 and 7) - These figures show that if different thresholds can be used, there will be more flexibility in selecting the proper thresholds to achieve the desired detection sensitivity. For example, the same detection sensitivity of $85.6 \%$ achieved by using $0.85 \mathrm{~mm}$ for both leads shown in Figure 4 can also be obtained by using a pair of different thresholds: $0.85 \mathrm{~mm}$ and $0.90 \mathrm{~mm}$, or $0.70 \mathrm{~mm}$ and $0.95 \mathrm{~mm}$.

Any three leads (Figure 8) - As expected, requiring three leads to exceed the threshold for an ST-alarm further reduces the detection sensitivity.

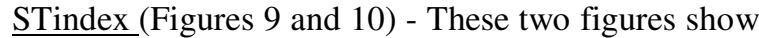
that the STindex computed from leads III, V2, and V5 is a better choice for ST-alarm detection. This is because (1) it has a much smaller detection variation for different occluded vessels, and (2) it uses a higher detection threshold of $1.5 \mathrm{~mm}$ (as opposed to $1.3 \mathrm{~mm}$ for leads aVF, V2, and V5) to achieve the same single-lead detection sensitivity obtained at a threshold of $1 \mathrm{~mm}$.

Performance summary (Figure 11) - This summary plot clearly shows that there is a noticeable performance drop between using any single lead and multiple leads. To match the single-lead sensitivity performance, a threshold of $1.5 \mathrm{~mm}$ should be used for the STindex (III, V2, V5). An even higher threshold of $2.0 \mathrm{~mm}$ can be used if the goal is to match the two-lead sensitivity performance.

\section{Conclusion}

Several ST-alarm strategies have been evaluated using a database of 12-lead ECGs recorded during PTCAinduced ischemia. The results suggest that using two leads (whether any two or any two contiguous leads using the same or different threshold values) for ST-alarm detection is a good compromise between maintaining high detection sensitivity and reducing false ST-alarms. The single-valued STindex computed from leads III, V2, and V5 provides the highest correlation to the 12-lead ST changes. The STindex can be used to achieve high sensitivity in ST-alarm detection, especially when monitoring using all 12 leads is not practical. These observations should be further validated using other ST databases as well as data obtained from real-time monitoring.

\section{References}

[1] Crawford MH, et al. ACC/AHA Guidelines for Ambulatory Electrocardiography. J Am Col Cardiol 1999;34:912-948.

[2] Drew BJ, Krucoff MW. Multilead ST-Segment Monitoring in Patients with Acute Coronary Syndromes: A Consensus Statement for Healthcare Professionals. Am J of Crit Care 1999;8:372-388.

[3] Wang JY. Noise Stress Testing for Real-Time ST-Segment Measurement Algorithms: A New Methodology. Computers in Cardiology 2000;27:845-848.

[4] Ryan TJ, et al. ACC/AHA Guidelines for the Management of Patients with Acute Myocardial Infarction. J Am Col Cardiol 1996; 28:1328-1428.

[5] Horáček BM, et al. Optimal Electrocardiographic Leads for Detecting Acute Myocardial Ischemia. J Electrocardiol 2001;34(Suppl):97-111.

[6] Mirvis DM, et al. Instrumentation and Practice Standards for Electrocardiographic Monitoring in Special Care Units. Circulation 1989;79:464-471.

Address for correspondence:

John Wang

Philips Medical Systems

3000 Minuteman Road, MS-0455

Andover, MA 01810-1099

USA

E-mail: john.j.wang@philips.com 